\title{
Comparison between laboratory measurements, simulations, and analytical predictions of the transverse wall impedance at low frequencies
}

\author{
F. Roncarolo, ${ }^{1,2}$ F. Caspers, ${ }^{3}$ T. Kroyer, ${ }^{3, *}$ E. Métral, ${ }^{3}$ N. Mounet, ${ }^{3,4}$ B. Salvant, ${ }^{3,4}$ and B. Zotter ${ }^{3}$ \\ ${ }^{1}$ School of Physics and Astronomy, The University of Manchester, Manchester M13 9PL, United Kingdom \\ ${ }^{2}$ The Cockcroft Institute, Daresbury WA4 4AD, United Kingdom \\ ${ }^{3}$ CERN, CH-1211 Geneva 23, Switzerland \\ ${ }^{4}$ EPFL, CH-1015 Lausanne, Switzerland
}

(Received 26 May 2009; published 26 August 2009)

\begin{abstract}
The prediction of the transverse wall beam impedance at the first unstable betatron line $(8 \mathrm{kHz})$ of the CERN Large Hadron Collider (LHC) is of paramount importance for understanding and controlling the related coupled-bunch instabilities. Until now only novel analytical formulas were available at this frequency. Recently, laboratory measurements and numerical simulations were performed to cross-check the analytical predictions. The experimental results based on the measurement of the variation of a probe coil inductance in the presence of (i) sample graphite plates, (ii) stand-alone LHC collimator jaws, and (iii) a full LHC collimator assembly are presented in detail. The measurement results are compared to both analytical theories and simulations. In addition, the consequences for the understanding of the LHC impedance are discussed.
\end{abstract}

DOI: 10.1103/PhysRevSTAB.12.084401

PACS numbers: 41.20.- q, 92.05.Bc, 29.20.D-, 41.75.-i

\section{INTRODUCTION}

When calculating resistive-wall impedances of particle accelerator components, in the case of poorly conductive materials, beams very close to the component wall and for frequencies low enough to have skin depths comparable to, or larger than, the wall thickness, novel theories (see [1] and included references) differ from the classical thickwall prediction. In particular, while the classical theory predicts the real and the imaginary part of the transverse impedance increasing with $1 / \sqrt{f}$ (where $f$ is the frequency) when going to low frequencies, more recent calculations estimate, below a certain frequency which depends on geometry and material conductivity, a decreasing real part (down to 0 at dc) and a constant imaginary part. This is why this effect is sometimes referred to as "inductive by-pass effect" [2] or "redistribution of the image currents effect" [3].

\section{ANALYTICAL PREDICTION}

The resistive-wall impedance of cylindrical vacuum chambers was first calculated more than 40 years ago under some approximations [4]. Since then many papers have been published to extend its range of validity. In the past few years, the interest in this subject has again been revived for the LHC graphite collimators, for which a new physical regime is predicted [1,2,5-11]. Indeed, the numerous col-

\footnotetext{
*Present address: Fraunhofer Institut Solare Energiesysteme, Freiburg, Germany.
}

limators in the machine are made of highly resistive graphite to withstand the high temperatures generated by the impact of high-energy protons, and will be moved into positions very close to the beam to protect the surrounding superconducting magnets from stray protons. These devices could create such high transverse resistive-wall impedances as to severely limit the beam current and hence the performance of the collider. The first unstable betatron line in the LHC is at $8 \mathrm{kHz}$, where the skin depth for graphite is $1.8 \mathrm{~cm}$, which is smaller than the collimator thickness of $2.5 \mathrm{~cm}$. Hence, one could think that the resistive thick-wall formula would be about right. It is found that it is not, and that the resistive impedance is about 2 orders of magnitude lower at this frequency, which is explained by the fact that the skin depth is much larger than the beam pipe radius. Consequently, the induced currents are further away (in average) from the beam, compared to the case where the skin depth is much smaller than the beam pipe radius.

Starting from the Maxwell equations and using field matching, a consistent derivation of the transverse "wall impedance" of an infinitely long cylindrical beam pipe was obtained. The results, which should be valid for any number of layers, beam velocity, frequency, conductivity, permittivity, and permeability, have been compared to previous ones. It is worth mentioning that the lowfrequency regime, which is of primary importance for the LHC collimators, was already obtained in [12] under some approximations. Furthermore, it should also be mentioned that the longitudinal impedance was derived as well [1], 


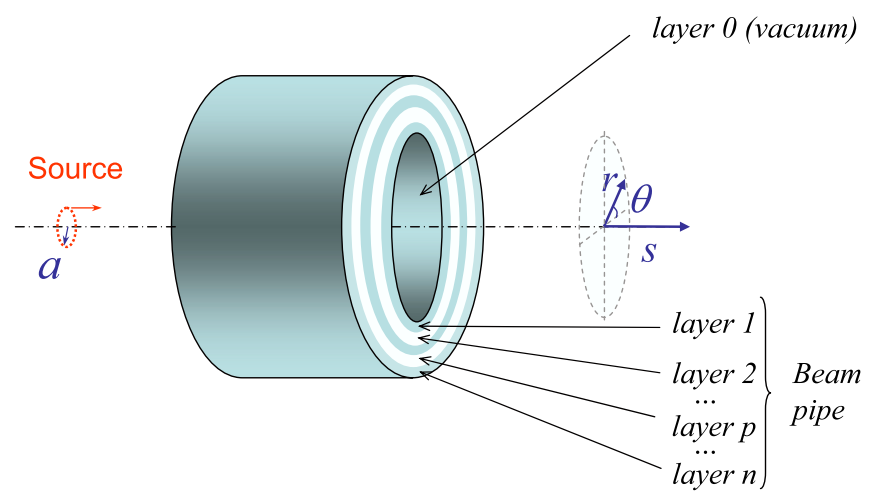

FIG. 1. (Color) Geometry of the beam pipe.

revealing also a different regime at low frequencies, but it will not be discussed in the present paper devoted to the transverse plane.

\section{A. Fields calculation}

We consider a cylindrical structure with infinite length, composed of several concentric layers of homogeneous materials (see Fig. 1). Starting from Maxwell equations in the frequency domain, where time derivatives are replaced by $j \omega$, and combining the conduction and displacement current terms, yields the following scalar Helmholtz equations in the circular cylindrical coordinates $(r, \theta, s)$ for the longitudinal field components in any of the concentric layers:

$$
\begin{gathered}
{\left[\frac{1}{r} \frac{\partial}{\partial r}\left(r \frac{\partial}{\partial r}\right)+\frac{1}{r^{2}} \frac{\partial^{2}}{\partial \theta^{2}}+\frac{\partial^{2}}{\partial s^{2}}+\omega^{2} \mu \varepsilon_{c}\right] H_{s}=0,} \\
{\left[\frac{1}{r} \frac{\partial}{\partial r}\left(r \frac{\partial}{\partial r}\right)+\frac{1}{r^{2}} \frac{\partial^{2}}{\partial \theta^{2}}+\frac{\partial^{2}}{\partial s^{2}}+\omega^{2} \mu \varepsilon_{c}\right] E_{s}} \\
=\frac{1}{\varepsilon_{0} \varepsilon_{b}} \frac{\partial \rho}{\partial s}+j \omega \mu \rho v .
\end{gathered}
$$

Here, $H_{s}$ and $E_{s}$ are the longitudinal magnetic and electric fields respectively, $\omega=2 \pi f$ is the angular frequency, $j$ is the imaginary unit, $\rho$ is the (source) charge density, $v$ is the source velocity (that we assume to be along the $s$ axis), and

$$
\begin{gathered}
\varepsilon_{c}=\varepsilon_{0} \varepsilon_{1}=\varepsilon_{0}\left(\varepsilon_{r}^{\prime}-j \varepsilon_{r}^{\prime \prime}\right)=\varepsilon_{0} \varepsilon_{b}+\frac{\sigma}{j \omega}, \\
\mu=\mu_{0} \mu_{1}=\mu_{0} \mu_{r}\left[1-j \tan \left(\vartheta_{M}\right)\right],
\end{gathered}
$$

where $\varepsilon_{c}(\mu)$ is the complex permittivity (permeability) of the medium in the layer considered, $\varepsilon_{1}\left(\mu_{1}\right)$ its relative complex permittivity (permeability), and $\varepsilon_{0}\left(\mu_{0}\right)$ the permittivity (permeability) of vacuum. $\mu_{r}$ is the real part of the relative complex permeability and $\tan \left(\vartheta_{M}\right)$ is the magnetic loss tangent. $\varepsilon_{1}$ can be written in terms of its real and imaginary parts $\varepsilon_{r}^{\prime}$ and $-\varepsilon_{r}^{\prime \prime}$, or equivalently in terms of the "normal" (and real) dielectric constant $\varepsilon_{b}$ and the electric conductivity $\sigma$ [13]. In the most general case, $\sigma$ depends on $\omega$ and contributes both to the real and imaginary parts of $\varepsilon_{1}$. We use in this section an ac complex conductivity,

$$
\sigma=\frac{\sigma_{\mathrm{dc}}}{1+j \omega \tau},
$$

where $\sigma_{\mathrm{dc}}=\rho_{\mathrm{dc}}^{-1}$ is the dc conductivity of the pipe and $\tau$ its relaxation time.

Note that in Eq. (2), in the charge density $\rho$ one should take into account an additional surface charge density at each interface between adjacent layers, due to the discontinuity in the conduction currents. Such a surface charge density does not play a role in the wave equations in the bulk of the layer but has an impact on the matching conditions perpendicularly to each interface, as $\varepsilon_{0} \varepsilon_{b} E_{r}$ will be discontinuous from an amount corresponding to the surface density. Alternatively, we could have written Gauss's law in terms of the full complex permittivity $\varepsilon_{c}$, as done in [14], resulting in a simpler radial matching condition as no surface charge needs then to be taken into account.

The homogeneous equations can be solved by separation of variables, writing the longitudinal component of the magnetic and electric fields as $\Theta(\theta) S(s) R(r)$. It is found that $\Theta(\theta)=e^{ \pm j m \theta}$, where $m$ is called the azimuthal mode number, and $S(s)=e^{ \pm j k s}$, where $k$ is called the wave number. The function $R(r)$ is found by solving the following equation:

$$
\frac{1}{r} \frac{d}{d r}\left(r \frac{d R}{d r}\right)-\left(\frac{m^{2}}{r^{2}}+\nu^{2}\right) R=0
$$

with $\nu=k \sqrt{1-\beta^{2} \varepsilon_{1} \mu_{1}}$, which is called the radial propagation constant, where $\beta$ is the relativistic velocity factor. It is known that the solutions of the differential Eq. (6) are the modified Bessel functions of $m$ th order and argument $\nu r$, called $I_{m}(\nu r)$ and $K_{m}(\nu r)$. The source charge density $\rho$ has to be specified at this stage to obtain the exact forms of the azimuthal and axial functions $\Theta(\theta)$ and $S(s)$. A macroparticle of charge $Q=N_{b} e$ is assumed to move along the pipe (in the $s$ direction) with an offset $r=a$ (assumed to be nonzero) in the $\theta=0$ direction and with velocity $v=\beta c$ (equal to the bunch velocity $v_{b}=\beta_{b} c$ ).

Neglecting betatron and synchrotron oscillations, assuming that the beam is not affected by the wakefield, and using the azimuthal Fourier decomposition of the charge, the charge density can be written as [15]

$$
\rho(r, \theta, s ; t)=\sum_{m=0}^{\infty} \frac{P_{m} \cos (m \theta)}{\pi a^{m+1}\left(1+\delta_{m 0}\right)} \delta(r-a) \delta(s-v t),
$$

where $P_{m}=Q a^{m}$ is the $m$ th multipole moment, and $\delta_{m 0}=1$ if $m=0,0$ if $m \neq 0$. Proceeding to the frequency domain and considering only one mode $m$, it is seen that the charge density $\rho_{m}$ is proportional to $\cos (m \theta) e^{-j k s}$ with $k=\omega / v$, which leads to the following longitudinal com- 
ponents of the electric and magnetic fields in any layer:

$$
\begin{aligned}
& H_{s}=\sin (m \theta) e^{-j k s}\left[C_{1} I_{m}(\nu r)+C_{2} K_{m}(\nu r)\right], \\
& E_{s}=\cos (m \theta) e^{-j k s}\left[C_{3} I_{m}(\nu r)+C_{4} K_{m}(\nu r)\right],
\end{aligned}
$$

where $C_{1,2,3,4}$ are constants (with different values for each layer) to be determined by field matching, as all field strength components have to be matched, i.e., in the absence of surface currents the four tangential field strengths have to be continuous at each interface between adjacent layers, except at $r=a$ (matching of the radial components is less straightforward, as seen above, and anyway redundant). Note that in the region where $r \leq a$ we get $C_{2}=$ $C_{4}=0$ in the above expressions of $H_{s}$ and $E_{s}$ as the $K_{m}$ modified Bessel functions go to infinity for $r=0$. The matching condition at $r=a$ is also different due to the presence of the beam and was derived in [16].

Finally, using Maxwell equations in a source-free region, the transverse field components in each layer can be deduced from the longitudinal ones (with $\mathbf{G}=Z_{0} \mathbf{H}$, where $Z_{0}$ is the free-space impedance)

$$
\begin{gathered}
E_{r 0}=\frac{j k}{\nu^{2}}\left(\beta \mu_{1} \frac{m G_{s 0}}{r}+\frac{d E_{s 0}}{d r}\right), \\
E_{\theta 0}=-\frac{j k}{\nu^{2}}\left(\frac{m E_{s 0}}{r}+\beta \mu_{1} \frac{d G_{s 0}}{d r}\right), \\
G_{r 0}=\frac{j k}{\nu^{2}}\left(\beta \varepsilon_{1} \frac{m E_{s 0}}{r}+\frac{d G_{s 0}}{d r}\right), \\
G_{\theta 0}=\frac{j k}{\nu^{2}}\left(\frac{m G_{s 0}}{r}+\beta \varepsilon_{1} \frac{d E_{s 0}}{d r}\right),
\end{gathered}
$$

where $E_{s} / E_{s 0}=E_{r} / E_{r 0}=G_{\theta} / G_{\theta 0}=\cos (m \theta)$ and $E_{\theta} / E_{\theta 0}=G_{r} / G_{r 0}=G_{s} / G_{s 0}=\sin (m \theta)$. Note that in these equations, $\varepsilon_{1}, \mu_{1}$, and $\nu$ have different values depending on the region where the equation is applied: in each layer of the pipe wall these quantities are defined according to Eqs. (3) and (4) whereas in vacuum $\mu_{1}$ and $\varepsilon_{1}$ should be replaced by 1 and $\nu$ by $k / \gamma$, where $\gamma=(1-$ $\left.\beta^{2}\right)^{-1 / 2}$ is the relativistic mass factor. The same remark holds for $\varepsilon_{b}, \varepsilon_{c}$, and $\mu$ in Eqs. (1) and (2).

\section{B. General formula for the transverse impedance}

Considering only the term $m=1$ (transverse dipole), the charge density in the frequency domain is given by

$$
\rho_{1}(r, \theta, s ; \omega)=\frac{P_{1}}{\pi a^{2} v} \delta(r-a) \cos \theta e^{-j k s} .
$$

The longitudinal source-field components (for $a \leq r \leq b$, where $b$ is the inner beam pipe radius) have been computed in [16] and are given by

$$
\begin{aligned}
E_{s}(r, \theta, s ; \omega)= & \frac{j \omega P_{1}}{\pi a \varepsilon_{0} v^{2} \gamma^{2}} I_{1}\left(x_{0}\right) \\
& \times \cos \theta\left[K_{1}(u)-\alpha_{\mathrm{TM}} I_{1}(u)\right] e^{-j k s,} \\
G_{s}(r, \theta, s ; \omega) & =\frac{j \omega P_{1}}{\pi a \varepsilon_{0} v^{2} \gamma^{2}} I_{1}\left(x_{0}\right) \sin \theta \alpha_{\mathrm{TE}} I_{1}(u) e^{-j k s,}
\end{aligned}
$$

where $x_{0}=k a / \gamma, u=k r / \gamma$, and the unknown parameters $\alpha_{\mathrm{TM}}$ and $\alpha_{\mathrm{TE}}$ have to be found by field matching at the layers' boundaries. The total horizontal impedance is given by

$$
\begin{aligned}
Z_{x}^{\text {Total }}(\omega)= & \frac{j}{P_{1}} \int_{-\infty}^{+\infty} d s\left[E_{x}-v_{b} B_{y}\right] e^{j k s} \\
= & \frac{j}{P_{1}} \int_{-\infty}^{+\infty} d s\left[E_{\theta}\left(a,-\frac{\pi}{2}, s ; \omega\right)\right. \\
& \left.+v_{b} B_{r}\left(a,-\frac{\pi}{2}, s ; \omega\right)\right] e^{j k s},
\end{aligned}
$$

which, using Eqs. (11) and (12), yields (with $L$ the length of the object)

$$
Z_{x}^{\mathrm{total}}(\omega)=-\frac{j L Z_{0} I_{1}\left(x_{0}\right) K_{1}\left(x_{0}\right)}{\pi a^{2} \beta \gamma^{2}}+\alpha_{\mathrm{TM}} \frac{j L Z_{0} I_{1}^{2}\left(x_{0}\right)}{\pi a^{2} \beta \gamma^{2}} .
$$

The wall impedance (and not the resistive-wall impedance) is obtained by subtracting from the total impedance of Eq. (18), the incoherent part of the impedance [i.e. which does not depend on the wall, and comes from the direct space charge (SC) interaction] given by

$$
Z_{x}^{\mathrm{SC}, \text { incoh }}(\omega)=-\frac{j L Z_{0} I_{1}\left(x_{0}\right) K_{1}\left(x_{0}\right)}{\pi a^{2} \beta \gamma^{2}} .
$$

The present formalism can also be used for any number of layers of the vacuum pipe [9]. Only the result for a single layer extending up to infinity is given here:

$$
\begin{aligned}
Z_{x}^{\text {wall }}(\omega)= & \frac{j L Z_{0} I_{1}^{2}\left(x_{0}\right) K_{1}\left(x_{1}\right)}{\pi a^{2} \beta \gamma^{2} I_{1}\left(x_{1}\right)}+j L Z_{0} \beta I_{1}^{2}\left(x_{0}\right) K_{1}\left(x_{1}\right) x_{1}^{2} x_{2}^{2} \nu\left(\frac{I_{1}^{\prime}\left(x_{1}\right)}{I_{1}\left(x_{1}\right)}-\frac{K_{1}^{\prime}\left(x_{1}\right)}{K_{1}\left(x_{1}\right)}\right)\left(\gamma \nu \frac{I_{1}^{\prime}\left(x_{1}\right)}{I_{1}\left(x_{1}\right)}-k \mu_{1} \frac{K_{1}^{\prime}\left(x_{2}\right)}{K_{1}\left(x_{2}\right)}\right) / \\
& \left\{\pi a^{2} \gamma I_{1}\left(x_{1}\right)\left[\left(\gamma \nu x_{2}-k x_{1}\right)^{2}-\left(\beta x_{1} x_{2}\right)^{2}\left(\gamma \nu \frac{I_{1}^{\prime}\left(x_{1}\right)}{I_{1}\left(x_{1}\right)}-k \mu_{1} \frac{K_{1}^{\prime}\left(x_{2}\right)}{K_{1}\left(x_{2}\right)}\right)\left(\gamma \nu \frac{I_{1}^{\prime}\left(x_{1}\right)}{I_{1}\left(x_{1}\right)}-k \varepsilon_{1} \frac{K_{1}^{\prime}\left(x_{2}\right)}{K_{1}\left(x_{2}\right)}\right)\right]\right\},
\end{aligned}
$$




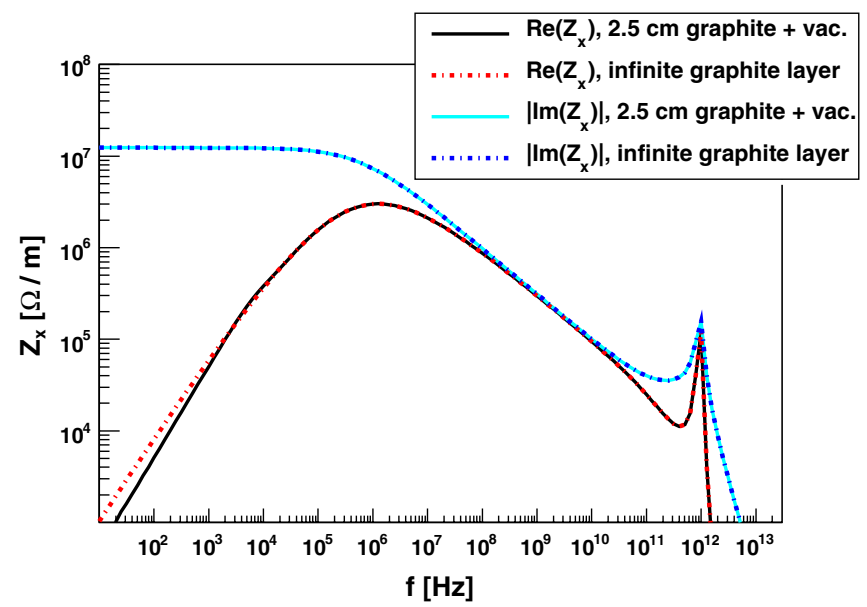

FIG. 2. (Color) Real and imaginary transverse wall impedance for the case of an LHC graphite collimator at injection energy with $b=2 \mathrm{~mm}, \rho_{\mathrm{dc}}=10 \mu \Omega \mathrm{m}, \tau=0.8 \mathrm{ps}, \varepsilon_{b}=1, L=$ $1 \mathrm{~m}, \gamma=480, a=10 \mu \mathrm{m}, \mu_{r}=1$, and $\tan \left(\vartheta_{M}\right)=0$, assuming $2.5 \mathrm{~cm}$ jaws surrounded by vacuum (solid black and cyan lines) and infinitely thick graphite jaws (dashed red and blue lines). Note that the (dipolar) Yokoya factor for a flat chamber $\left(\pi^{2} / 12\right)$ has been applied.

where $x_{1}=k b / \gamma$ and $x_{2}=\nu b$. The transverse wall impedance as calculated for a LHC graphite collimator is shown in Fig. 2. The different curves refer to real and imaginary part of the transverse impedance in the case of a single layer of graphite extending to infinity and of a $2.5 \mathrm{~cm}$ thick graphite layer surrounded by an infinite volume of vacuum. It is evident that the difference between these two cases is noticeable only for the real part of the impedance at frequencies below $1 \mathrm{kHz}$.

In addition, Fig. 2 shows three frequency regimes: (1) A "low-frequency" one, up to $1 \mathrm{MHz}$ in the present case, (2) an "intermediate-frequency" one, where the classical thick-wall formula is valid, and (3) a "high-frequency" one, which is discussed in [17], but which is not relevant for the LHC. For all three regimes, a comparison between the curves of Fig. 2 and the theory of Henry and Napoly [12] can be found in [1], whereas [10] contains a comparison with Burov-Lebedev [5] in the first two regimes and with Bane [17] in the third one. All the comparisons exhibit an excellent agreement for the case of an LHC collimator.

Note that in the case of a noncylindrical structure, Yokoya [18] (or Laslett [19]) factors should be applied. This means that for instance in the case of two horizontal parallel plates (or a rectangular structure in which the width is much larger than the height), the horizontal impedance is deduced from the round one by applying the factor $\pi^{2} / 24$, while it is $\pi^{2} / 12$ in the vertical plane. Furthermore, in addition to these (dipolar) impedances, quadrupolar (also called detuning or incoherent) impedances are also introduced. They can also be deduced from the round (dipolar) impedance by applying the factors $-\pi^{2} / 24$ in the horizontal plane and $\pi^{2} / 24$ in the vertical

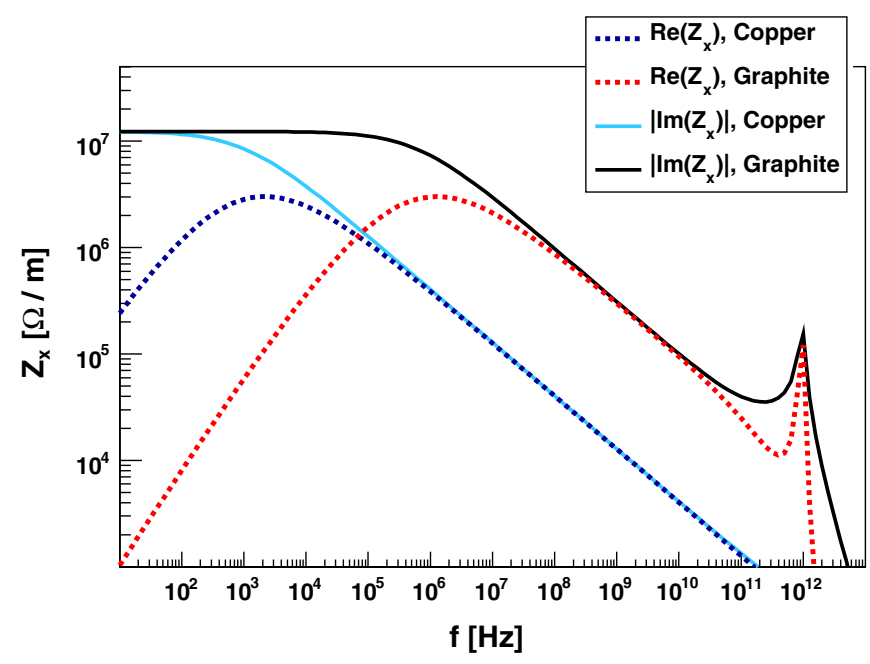

FIG. 3. (Color) Real and imaginary transverse wall impedance for the case of an LHC collimator at injection energy with $b=$ $2 \mathrm{~mm}$ assuming infinitely thick jaws of copper (dashed blue and solid cyan lines) and of graphite (dashed red and solid black lines). The other parameters are those of Fig. 2, except for the dc resistivity and relaxation time of copper: $\rho_{\mathrm{dc}}=1.7 \times 10^{-8} \Omega \mathrm{m}$ and $\tau=2.7 \times 10^{-14} \mathrm{~s}$.

one. The real and imaginary part of the transverse wall impedance as calculated for LHC collimatorlike structures in graphite and copper are shown in Fig. 3. This figure shows that the maximum amplitude of the real impedance does not depend on the material conductivity but the frequency at which the maximum occurs does, as it will be discussed in the next section. As a consequence, at low frequencies copper has a higher real impedance than graphite. The figure also reveals that the constant value at which the imaginary impedance tends for $f$ approaching the dc regime does not depend on the material conductivity.

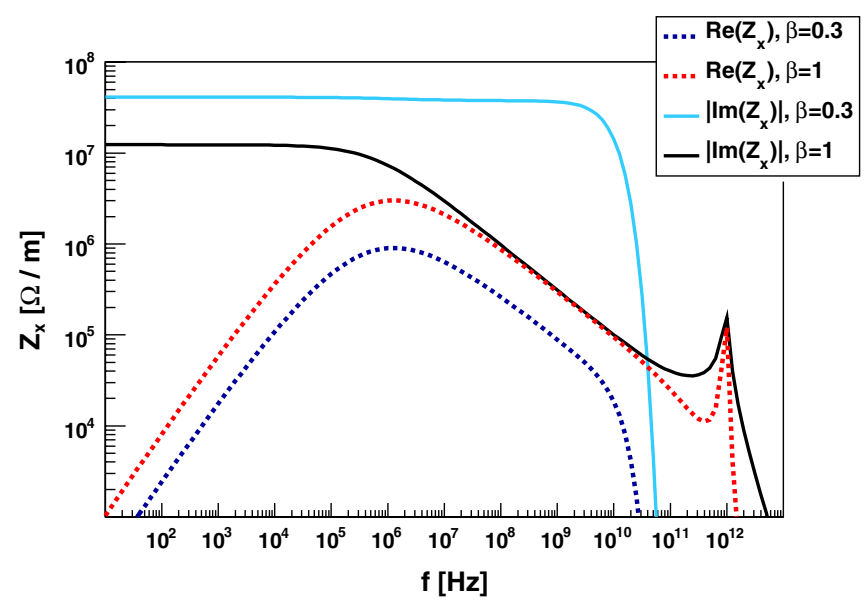

FIG. 4. (Color) Transverse wall impedance for the case of an LHC collimator with $b=2 \mathrm{~mm}$ assuming infinitely thick graphite jaws with $\beta=0.3$ (dashed blue and solid cyan lines) and $\beta=1$ (dashed red and solid black lines). The other parameters are those of Fig. 2. 
On the other hand, such asymptotic value depends on the relativistic factor $\beta$ as shown in Fig. 4. The lower $\beta$ the higher the dc imaginary impedance and the lower the maximum real impedance.

Even if the effect of $\beta$ on the wall impedance is not relevant for the case of LHC collimators, a few more details are presented in the Appendix.

\section{Approximate formula for an LHC (graphite) collimator}

The interesting frequency range in the LHC lies between few $\mathrm{kHz}$ and few $\mathrm{GHz}$. In this case a much simpler formula than the one given in Eq. (20) can be derived for a cylindrical geometry, which should be valid for any "relatively" good conductor ( $\varepsilon_{r}^{\prime}$ small compared to $\left.\varepsilon_{r}^{\prime \prime}\right)$ with real permeability $\left(\mu_{1}=\mu_{r}\right)$. In a frequency range with upper limit depending on $\beta$ [11], it can be written as

$Z_{x}^{\text {wall }}(\omega) \approx \frac{j L Z_{0} I_{1}^{2}\left(x_{0}\right) K_{1}\left(x_{1}\right)}{\pi a^{2} \beta \gamma^{2} I_{1}\left(x_{1}\right)}+\beta \frac{j L Z_{0}}{\pi b^{2}} \times \frac{1}{1-\frac{x_{2}}{\mu_{r}} \times \frac{K_{1}^{\prime}\left(x_{2}\right)}{K_{1}\left(x_{2}\right)}}$,

where

$$
x_{2}=(1+j) \frac{b}{\delta} \text { and } \delta=\sqrt{\frac{2}{\mu_{0} \mu_{r} \sigma \omega}} .
$$

Furthermore, assuming $\mu_{r}=1$, using $K_{1}(x) \approx 1 / x$, $I_{1}(x) \approx x / 2$ for $|x| \ll 1$ and the following equation,

$$
\frac{K_{1}^{\prime}\left(x_{2}\right)}{K_{1}\left(x_{2}\right)} \approx \mid \begin{array}{cc}
-\frac{1}{x_{2}} & \text { if }\left|x_{2}\right| \ll 1 \\
-1 & \text { if }\left|x_{2}\right| \gg 1
\end{array},
$$

Eq. (21) can be simplified even further in the two limiting cases specified in the next paragraphs.

When $\left|x_{2}\right| \ll 1$, i.e., at very low frequency, the transverse wall impedance approaches a constant inductive value,

$$
Z_{x}^{\text {wall }}(\omega) \underset{\omega \rightarrow 0}{\longrightarrow} \frac{j \succeq Z_{0}}{2 \pi \beta b^{2}}\left(1-\beta^{2}\right)+\beta \frac{j L Z_{0}}{2 \pi b^{2}}=\frac{j L Z_{0}}{2 \pi \beta b^{2}},
$$

and its numerical value in the case of the parameters of Fig. 2 is $12.3 j \mathrm{M} \Omega / \mathrm{m}$ (applying the Yokoya factor of $\pi^{2} / 12$ ). Note that with Eq. (24), the impedance formula of a conducting beam pipe in the dc regime is recovered (when only the coherent part is taken into account): only the contribution from the electric images (the term 1 in the parenthesis) remains; the contribution from the ac magnetic images (the term $-\beta^{2}$ in the parentheses) disappears as expected at very low frequencies in a conductor. Note that this will not be the case in the second frequency regime, discussed in the next paragraph, where the socalled coherent space-charge impedance (with contribution from both electric and ac magnetic images) is separated from the usual resistive-wall impedance (i.e. coming from the resistivity of the vacuum pipe). Finally, it should be stressed that the imaginary part of the wall impedance increases with the inverse of $\beta$, i.e., the lower the beam energy the higher the wall impedance. When further developing the ratio $\frac{K_{1}^{\prime}\left(x_{2}\right)}{K_{1}\left(x_{2}\right)}$ around zero, one can also get the real part of the transverse wall impedance,

$$
\operatorname{Re}\left[Z_{x}^{\text {wall }}(\omega)\right]=-\frac{\beta L Z_{0}}{2 \pi \delta^{2}}\left[\ln \left(\frac{b}{\delta \sqrt{2}}\right)+\gamma_{e}\right]
$$

where $\gamma_{e} \approx 0.577$ is the Euler constant. We see that the real part of the impedance is not linear in $\omega$ : its leading term is proportional to $-\omega \ln \omega$. Indeed, the real part of the impedance decreases with $\omega$ as the contribution from the ac magnetic images decreases. On the other hand, the magnetic images penetrate more in the wall with decreasing frequency as the skin depth $\delta$ increases, and since $\delta \gg$ $b$ the distance that matters (i.e. between the beam and the average induced currents) is not $b$ anymore but $\propto \delta$, thus the $1 / \delta^{2}$ factor in Eq. (25), similar to the $1 / b^{2}$ factor in Eq. (24). We also see in Eq. (25) that the real part of the wall impedance increases with the conductor's conductivity: the higher $\sigma$, the nearer the currents will be to the beam because of the skin depth, so the higher the impedance. It can be seen in Fig. 5 that Eq. (25) perfectly matches the exact expression from Eq. (20), and matches quite well (in the frequency range of interest, that is, above $8 \mathrm{kHz}$ ) a two layers formula assuming $2.5 \mathrm{~cm}$ of graphite surrounded by vacuum, while it clearly deviates from a fit of the latter with an expression proportional to $\omega$.

When $\left|x_{2}\right| \gg 1$, i.e., at intermediate frequencies (up to a frequency which depends on $\beta$ ), the classical thick-wall

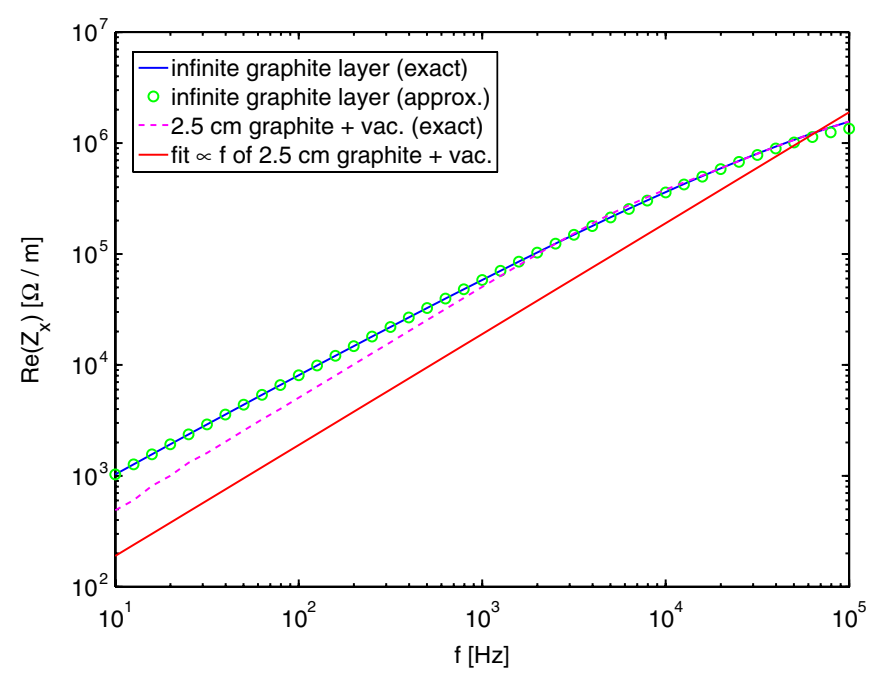

FIG. 5. (Color) Real part of the transverse wall impedance at low frequencies for the case of an LHC collimator with $b=2 \mathrm{~mm}$. We compare the exact expression for an infinitely thick graphite layer from Eq. (20) (solid blue line), its approximation by Eq. (25) (green circles), the exact expression for a $2.5 \mathrm{~cm}$ graphite layer surrounded by vacuum (dashed magenta line), and its fit by an expression proportional to $f$ (solid red line). The parameters are those of Fig. 2. 
formula is recovered:

$$
Z_{x}^{\text {wall }}(\omega)=\frac{j L Z_{0}}{2 \pi b^{2} \beta \gamma^{2}}+(1+j) \beta \frac{L Z_{0} \delta}{2 \pi b^{3}} .
$$

In this case, the wall impedance is composed of two terms: the first derives from the so-called coherent space-charge impedance (which disappears at very high energy, due to the cancellation between the electric images and the ac magnetic images), while the second depends on the resistivity of the vacuum chamber (it vanishes for $\sigma$ infinite as $\delta$ then goes to zero), and is usually called the "resistivewall" impedance. Note that the (broad) maximum of the real part of the transverse impedance is reached when $\operatorname{Re}\left[x_{2}\right] \approx 1$, i.e. $\delta \approx b$, which means

$$
f_{\max , \operatorname{Re}} \approx \frac{\rho}{b^{2}} \times \frac{1}{\pi \mu_{0}},
$$

where $\rho=\sigma^{-1}$ is the resistivity of the layer. In the case of an LHC collimator geometry Eq. (27) yields $f_{\mathrm{max}, \mathrm{Re}} \approx$ $1 \mathrm{MHz}$ for graphite and $f_{\text {max,Re }} \approx 3 \mathrm{kHz}$ for copper, as also shown in Fig. 3 (produced using the complete formalism).

\section{NUMERICAL SIMULATIONS}

Classical numerical simulation codes that solve in the frequency or time domain typical problems of beam coupling impedance provide poor accuracy below $1 \mathrm{MHz}$, i.e., in the frequency regime of interest mentioned above. In particular, time domain computer codes for this type of problem would require a huge length of wake to be simulated in order to get meaningful low-frequency data. Often these computer codes assume a lossless boundary which would not be applicable in the present case since losses are essential to solve the boundary value problem, in particular, to get the correct image current distribution. However, there are frequency domain programs optimized in the lowfrequency domain (like for the design of nondestructive testing devices using eddy currents or the optimization of transformers) that are suitable for our purposes. One of these codes, Ansoft Maxwell@ [20], was used [21] to predict the real part of the resistive-wall impedance for a number of relevant cases in which classical and novel theories diverge. In this section, we focus on simulations of the real part of the transverse impedance of a cylindrical structure, that is compared to analytical predictions.

\section{A. Simulation method and setup}

The simulations are based on the representation of a particle beam traveling through a device under test (DUT) by a thin wire conductor. The transverse electromagnetic wave (TEM) in the resulting coaxial structure is very similar to the field induced by an ultrarelativistic charge.

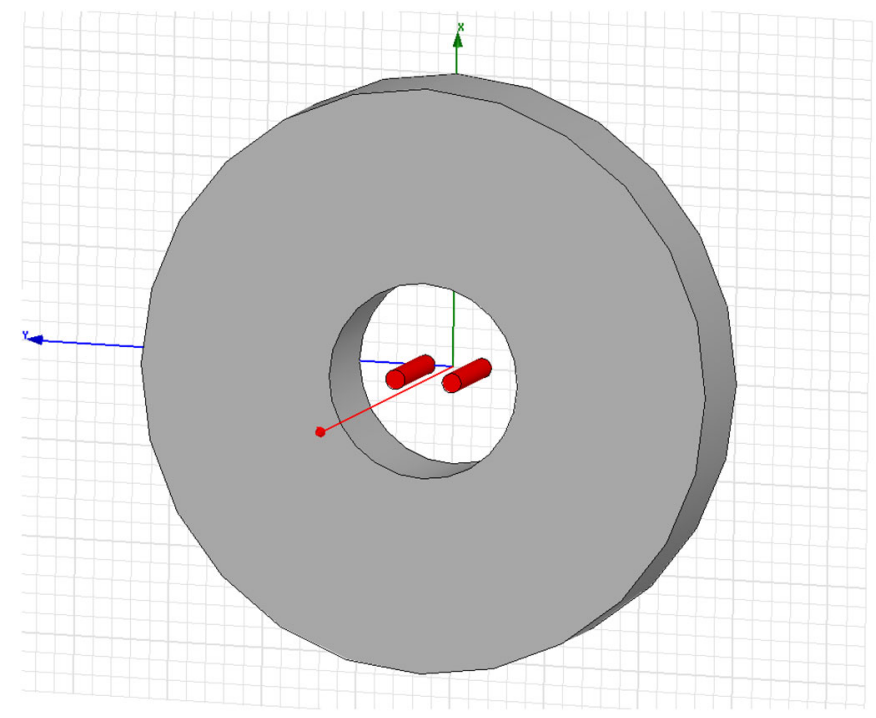

FIG. 6. (Color) 3D model used for the numerical simulations. A dipolar field is excited by the two wires (red) inside the lossy graphite cylinder (grey).

The DUT longitudinal impedance can be inferred by powering a single wire along the axis of the beam pipe. In a first order approximation, the real part of the longitudinal impedance $Z_{L}$ is proportional to the power lost in the DUT and the imaginary part to the phase shift of the wave. In general, the DUT transverse generalized (i.e. dipolar plus higher order modes) impedance $Z_{T}$ can be determined by calculating the variation of the longitudinal impedance for different off-axis wire positions. This method returns the sum of the dipolar and quadrupolar components of the transverse impedance. The dipolar term of the transverse impedance alone can be obtained using two wires in phase opposition, thus creating the dipolar field associated with the transverse impedance. The real part of $Z_{T}$ is still proportional to the power lost and its imaginary part to the phase shift.

As an example, the 3D model used to simulate a cylindrical beam pipe is shown in Fig. 6. Outside the DUT a perfect electrical conductor (PEC) was modeled. The beam runs along the $s$ axis. Only a very thin slice of an infinitely long structure was used. Collimatorlike geometries and more details about the simulations setup, including the use of symmetry planes and boundary conditions, are discussed in [21].

\section{B. Computation of the real part of the transverse impedance}

While rf simulations, as in laboratory measurements, at high frequencies directly yield the network scattering parameters [22], for simulations at low frequencies the complex impedances can be determined starting from the full 3D electromagnetic fields determined by the simulation.

Considering two parallel wires, stretched through the DUT along the beam direction and powered with a current 
of equal peak intensity $\hat{I}$ and opposite phase, the real part of the longitudinal impedance $Z_{L}$ can be determined as [21]

$$
\operatorname{Re}\left[Z_{L}\right]=\frac{2 \delta P}{\hat{I}^{2}},
$$

where $\delta P$ is the power lost in the DUT, which, in the numerical simulations, is calculated by integration of the Ohmic losses over the volume of the DUT. The transverse impedance characteristic of the two wires setup is expressed according to

$$
Z_{T}(\omega)=\frac{c}{\omega} \frac{Z_{L}}{\Delta^{2} L}
$$

where $c$ is the speed of light, $\Delta$ the wires spacing, and $L$ the DUT length. This formulation is discussed by Nassibian and Sacherer in [23] and will be reconsidered in the laboratory measurement section below.

Combining Eqs. (28) and (29), the real part of the transverse impedance results:

$$
\operatorname{Re}\left[Z_{T}\right](\omega)=\frac{c}{\omega} \frac{2 \delta P}{\hat{I}^{2} \Delta^{2} L} .
$$

\section{Uncertainties}

As discussed in [21], for obtaining Eq. (28), it is necessary to approximate the DUT as a distributed impedance and use the so-called "log formula" that allows calculating the power attenuation along a homogeneous transmission line. For reference cases like the ones discussed here and in [21], such an approximation is considered sufficiently appropriate. In addition, for the evaluation of the transverse impedance with the two wires method, the error due to a finite wire spacing is small provided that $\Delta / d \leq 0.3$ (where $d$ is the DUT total aperture) [24]. This allows having most of the $H$ field power confined between the wires while still avoiding too small wires which are difficult to mesh.

\section{Simulation results}

The real part of the transverse impedance as computed by the numerical simulation for a $1 \mathrm{~m}$ long, $10 \mathrm{~mm}$ thick graphite pipe is shown in Fig. 7. The plot evidences the effect of placing a $30 \mathrm{~mm}$ vacuum layer between the DUT and the perfect conductor boundary. At low frequencies, the real part of the transverse impedance is about twice the one resulting by placing the perfect conductor boundary in direct contact with the graphite. This is due to the fact that more current flows in the lossy graphite when the PEC is farther from the beam.

The results are compared to the analytical prediction for the same geometry and material, and the agreement is within $1 \%$. Comparable simulations were carried out for collimatorlike structures with similar agreement with the analytical calculations and all results are reported in [21].

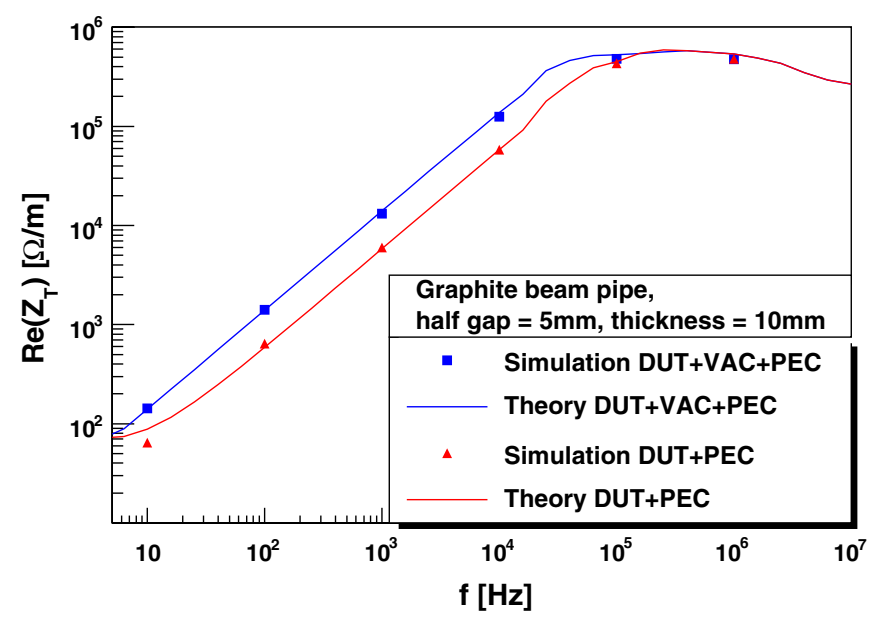

FIG. 7. (Color) Real part of the transverse impedance as calculated by numerical simulations [21] and analytical models [1] (cf. Sec. II) for cylindrical graphite beam pipes. PEC stands for perfect electrical conductor and VAC for vacuum. The effect of adding a $30 \mathrm{~mm}$ vacuum layer between the DUT and the PEC boundary is evident at low frequencies.

\section{E. Results interpretation}

In this section we give a physical interpretation of the numerical simulation results that concerns the real part of the transverse impedance. This is in good agreement with the interpretation of the analytical models presented in Sec. II.

The frequency dependence of the real part of the longitudinal impedance can be understood by an easy argument. We consider a beam (wire) going through an at least weakly conducting DUT embedded in a perfect conductor. The case of a DUT in free space corresponds to PEC at a very large distance. At dc all the currents flow in the PEC. There are no losses and the impedance is zero. For very low frequencies the beam-induced magnetic field in turn induces currents in the DUT according to Faraday's law,

$$
\vec{\nabla} \times \vec{E}=-\frac{\partial \vec{B}}{\partial t}
$$

The displacement currents can still be neglected. For harmonic excitation we get $E \propto f$, which gives rise to eddy currents with the same frequency dependence $J \propto f$. Then the losses $P \propto f^{2}$, which with Eq. (28) leads to $Z \propto f^{2}$. At very high frequencies all the current flows on the innermost layer of the DUT. Because of the skin effect the losses increase with $\sqrt{f}$ and so does $Z$.

For $Z_{T}$ the same argument is valid starting with a dipolar magnetic field. According to Eq. (29) $Z_{T} \propto Z / f$. Therefore at low frequencies $Z_{T} \propto f$ [more precisely, as was seen in Sec. II from Eq. (25), $\left.Z_{T} \propto f \ln f\right]$ and at high frequencies $Z_{T} \propto 1 / \sqrt{f}$. In between the two regimes $Z_{T}$ reaches its maximum value. 


\section{LABORATORY MEASUREMENTS}

The approach used for numerical simulations is not directly applicable to laboratory experiments due to the difficulty one can have in measuring the power loss in the DUT volume. Here we rediscuss the single and doublewire methods and, passing through Nassibian-Sacherer's theory [23] for a single loop, will introduce the "probe coil" method as the most suitable for the measurement of transverse wall impedances at low frequencies.

\section{A. Method}

A classical way for measuring the beam coupling impedance of an accelerator component consists in reproducing the electromagnetic interaction between the particles beam and the component by stretching a thin conductive wire along the reference beam trajectory inside the DUT and powering it with an rf source. Usually, a vector network analyzer is used as the rf source and at the same time allows measuring the scattering parameters of the resulting network, that, compared to similar measurements on a reference beam pipe, yields the DUT longitudinal impedance. The transverse (generalized) impedance is then calculated repeating the measurement at different wire transverse positions and looking at the variation of the longitudinal impedance. Theoretical and experimental aspects of this method are very well addressed by Vaccaro in [22] and Caspers in [25].

However, such a method has very poor sensitivity at low frequencies, for which the signals to be measured are extremely small.

An alternative method for transverse coupling impedances is based on a two-wire (or single loop) system. This is discussed in detail by Nassibian and Sacherer in [23] and in the following we review the basic steps.

Any beam that oscillates in a transverse coordinate induces electromagnetic fields in the surrounding materials. For the coherent part of the impedance, the electric field is neglected and only the magnetic field $\mathbf{B}$ is considered. This is true in the second frequency regime as can be seen for instance in Ref. [26] (where for the transverse components of the electromagnetic fields we have $E_{T} \propto$ $|z|^{-5 / 2}$ while $B_{T} \propto|z|^{-1 / 2},|z|=|s-v t|$ being the distance behind the beam), and in the first frequency regime this approximation holds only for the real part of the impedance (its imaginary part for $\omega \rightarrow 0$ is determined by the electric images, as was discussed in Sec. II). The transverse impedance then results:

$$
Z_{T}=\frac{j}{\hat{I} \Delta} \int_{0}^{L}(\mathbf{v} \times \mathbf{B})_{T} d s,
$$

where $\pm \Delta$ is the beam oscillation amplitude and $\hat{I}$ the beam current. Note that we have dropped the $\frac{1}{\beta}$ factor present in the original formula in [23], to be consistent with the impedance definition of Sec. II. The term that drives the correspondent force (acting back on the beam and potentially perturbing its stability) is the dipole moment $\Delta \cdot \hat{I}$.

The same effect occurs substituting the beam by two wires powered with opposite currents or by a wire loop of (unperturbed) impedance $Z_{0}$. In this case Eq. (32) gives the same result as Eq. (17) of Sec. II. The current density along the wires is indeed (in cylindrical coordinates)

$$
\begin{aligned}
J_{s}^{\mathrm{mes}} & =\frac{1}{a} \hat{I} \delta(r-a)[\delta(\theta)-\delta(\theta-\pi)] \\
& \approx \frac{2}{\pi a} \hat{I} \delta(r-a) \cos (\theta) .
\end{aligned}
$$

Higher order azimuthal modes are neglected here since their effects on the wall impedance involve Bessel functions $I_{m}\left(\frac{k a}{\gamma}\right)$ with $m>1$, which are negligible. $J_{s}^{\text {mes }}$ is proportional to the current density of Sec. II for the $m=$ 1 mode in the frequency domain, where (see also Refs. $[9,16])$

$$
J_{s}^{\mathrm{th}}=\rho v=\frac{P_{1}}{\pi a^{2}} \delta(r-a) \cos (\theta) e^{-j k s},
$$

in which the proportionality factor is equal to $\frac{2 a \hat{I}}{P_{1}} e^{j k s}$. The same factor should be applied for the resulting $\mathbf{B}$ field compared to the one of Sec. II. Here $\Delta=2 a$ is the distance between the two wires and consequently Eq. (32) gives the same impedance as Eq. (17) when neglecting the incoherent space-charge part.

The $\mathbf{B}$ field induced by the loop on the surroundings acts back generating a voltage on the loop,

$$
V=j \omega B L \Delta=Z_{B} \cdot \hat{I},
$$

where $L$ is the loop length and $Z_{B}$ is the consequent variation of the loop impedance. Measuring the total loop impedance $Z_{\text {meas }}$ allows calculating $Z_{B}=Z_{\text {meas }}-Z_{0}$ that according to Eqs. (32) and (35) gives the beam-induced field $B$ and the related transverse impedance as

$$
\begin{aligned}
& B=\frac{\hat{I} Z_{B}}{j \omega L \Delta}, \\
& Z_{T}=\frac{c Z_{B}}{\omega \Delta^{2}} .
\end{aligned}
$$

Also this method has poor sensitivity at low frequencies, but improved results can be achieved by substituting the two wires by a multiturn probe coil as proposed in [3]. The variation of the input coil impedance $Z_{\text {coil }}^{\mathrm{DUT}}$ in the presence of the DUT, compared to a reference measurement $Z_{\text {coil }}^{\text {ref }}$, gives the transverse beam coupling impedance associated to the DUT, according to

$$
Z_{T}^{\text {meas }}=\frac{c}{\omega} \frac{Z_{\text {coil }}^{\mathrm{DUT}}-Z_{\mathrm{coil}}^{\mathrm{ref}}}{N^{2} \Delta^{2}}
$$

where $N$ is the number of turns of the coil and $\Delta$ in this 
case is the transverse spacing of the coil wires. The comparison with a reference material by computing the difference $Z_{\text {coil }}^{\text {DUT }}-Z_{\text {coil }}^{\text {ref }}$ is meant to isolate the wall part of the DUT impedance. This is rigorous in the ideal case of having a measurement in free space as a reference. In practice, it is convenient to use as a reference high conductivity materials (like copper or brass) with the same DUT geometry.

Depending on the materials under test and the geometry (beam pipe diameter or collimator gap values), at very low frequencies the real part of the wall impedance of the reference material may well become larger than the one of the DUT and the measured quantity $Z_{T}^{\text {meas }}$ results negative. As an approximation, in all cases, one has to consider as exact the reference material wall impedance $Z_{W}^{\text {ref }}$ and retrieve the one of the DUT as

$$
Z_{W}^{\mathrm{DUT}}=Z_{T}^{\mathrm{meas}}+Z_{W}^{\mathrm{ref}}
$$

For most cases presented here, the predicted real part of the wall impedance of the reference material, down to frequencies of $1-2 \mathrm{kHz}$, depends very little from the used theory (classic thick wall or the one described in this paper). As an example, this can be seen in Fig. 3: the maximum of $\operatorname{Re}\left(Z_{x}\right)$ for copper, i.e., the boundary between classical and novel theories, is at about $1 \mathrm{kHz}$.

\section{B. Setup}

A number of laboratory experiments were carried out, reproducing in three different stages the geometry and material property conditions represented by the present LHC collimators, namely, (i) sample graphite plates, (ii) stand-alone LHC collimator jaws, and (iii) a full LHC collimator assembly. The geometry and material resistivity of the three measurement stages are summarized in Table I. Every time, copper with the same dimensions of the DUT and resistivity $\rho_{\mathrm{Cu}}=1.7 \times 10^{-8} \Omega \mathrm{m}$ was used as a reference. The DUT and reference material resistivity follow from dedicated measurements and were used for the analytical calculations (of this section only, since in Sec. II we used $\left.\rho_{c}=10 \mu \Omega \mathrm{m}\right)$.

Pictures of the three laboratory measurement setup stages are shown in Fig. 8. One of the most challenging

TABLE I. Geometry and material properties of the three different measurement stages (see text).

\begin{tabular}{|c|c|c|c|c|c|}
\hline \multirow[b]{2}{*}{ Stage } & \multicolumn{3}{|c|}{ Geometry } & \multirow[b]{2}{*}{ Material } & \multirow{2}{*}{$\begin{array}{c}\rho_{c} \\
{[\mu \Omega \mathrm{m}]}\end{array}$} \\
\hline & $L[\mathrm{~cm}]$ & $h[\mathrm{~cm}$ & $t[\mathrm{~cm}]$ & & \\
\hline 1 & 15 & 10 & 1 & Graphite & 13 \\
\hline 2 & 120 & 6.6 & 2.5 & Graphite & 13 \\
\hline 3 & $160^{\mathrm{a}}, 120^{\mathrm{b}}$ & 6.6 & 2.5 & $\begin{array}{l}\text { Carbon fiber-reinforced } \\
\text { carbon }(\mathrm{CFC})\end{array}$ & 5 \\
\hline
\end{tabular}

${ }^{\mathrm{a}}$ Collimator in which the CFC jaws are assembled.

${ }^{\mathrm{b}}$ Reference jaws and analytical calculations.
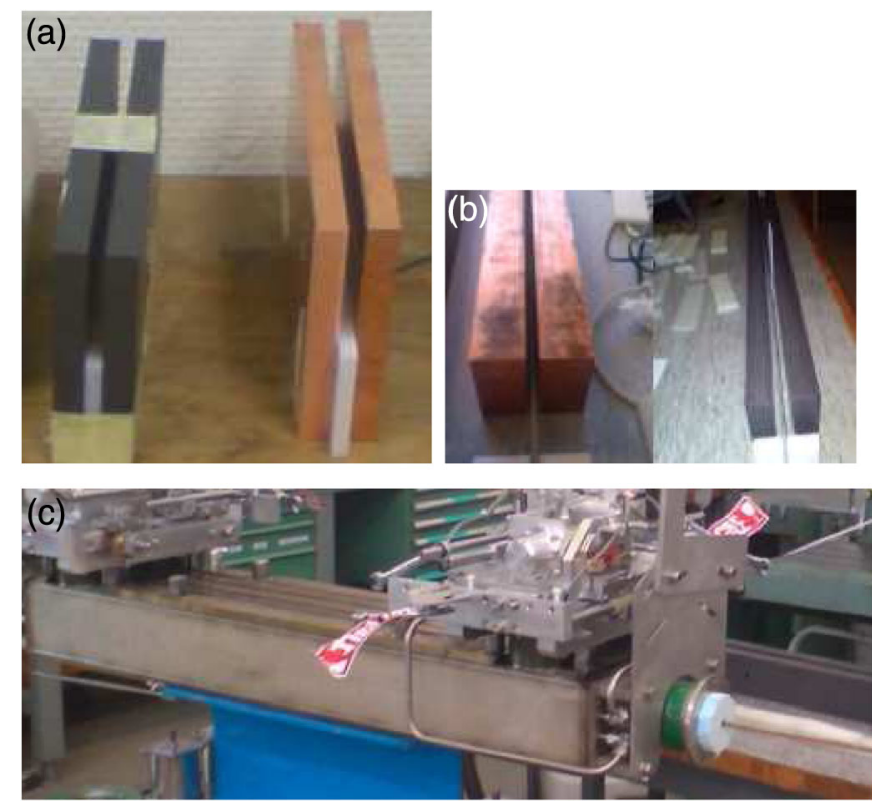

FIG. 8. (Color) Pictures of the three measurement setup stages: (a) sample plates $15 \mathrm{~cm} \times 10 \mathrm{~cm} \times 1 \mathrm{~cm}$, (b) stand-alone jaws $120 \mathrm{~cm} \times 10 \mathrm{~cm} \times 2.5 \mathrm{~cm}$, and (c) collimator assembly (jaws $120 \mathrm{~cm} \times 10 \mathrm{~cm} \times 2.5 \mathrm{~cm})$.

aspects of the measurements was related to the very small absolute value (smaller than $5 \Omega$ at low frequencies) and relative variation (down to fractions of $\mathrm{m} \Omega$ as will be shown in Fig. 9) of the relevant observable quantity (i.e. the input impedance of the probe coil). Initial tests based on the determination of the coil impedance by measuring the network scattering parameters with a Vector Network Analyzer (HP 8751A [27] or Agilent 4395A [28]) provided excellent results down to $10 \mathrm{kHz}$ but exhibited an unacceptable signal to noise ratio for lower frequencies. The noise figure resulted much smaller when using a LCR

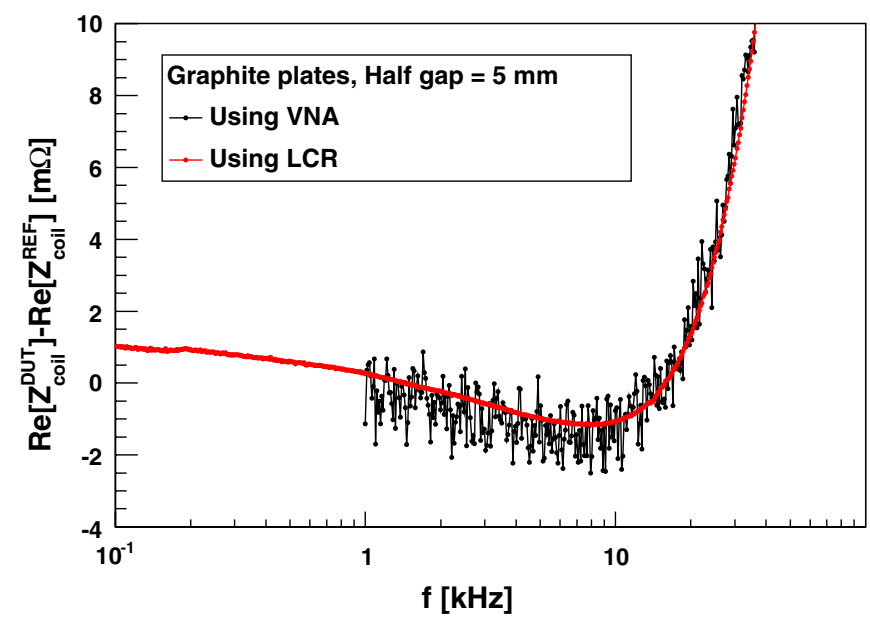

FIG. 9. (Color) Comparison of the signal to noise level while measuring the impedance of a probe coil with a VNA and with a LCR. The latter exhibits a much better noise figure. 
meter (Agilent E4980A [29]). This instrument operates only up to $2 \mathrm{MHz}$, but higher frequencies were not significant due to the occurrence of the first coil self-resonance for all the used coils. The comparison between a vector network analyzer (VNA) and a LCR noise levels while measuring the real part of the same probe coil inserted between two graphite plates is shown in Fig. 9. A GPIBLAN adapter (Tektronix AD007 [30]) was programmed to set, read out, and store the results of the LCR measurements with automatic procedures that optimized the measurement time.

\section{Uncertainties}

For each measurement stage at least two different probe coils were fabricated, differing in length $L$, number of turns $N$, and width $\Delta$. Each probe coil has been fabricated winding a $0.5 \mathrm{~mm}$ diameter copper wire around a rigid Fiberglass bar (the thickness of which determines the parameter $\Delta$ ). Typical parameters were $\Delta \geq 2.5 \mathrm{~mm}$ and $5 \leq N \leq 14$.

The probe coil design has a direct impact on the measurement accuracy. In particular: (i) The minimum coil length $L$ is constrained by the DUT length. Longer coils allow higher sensitivity (i.e. higher coil impedance and, consequently, a better signal to noise ratio) and facilitate the coil placement and alignment inside the DUT. On the other hand, each portion of the coil outside the DUT is affected by external fields, potentially variable between DUT and reference measurements. (ii) The smaller the coil width $\Delta$ the lower the coil impedance and, consequently, the lower the method sensitivity. However, only with small $\Delta$ it is possible to measure DUTs like small gap collimators. Indeed, it was possible to measure small gaps only at the cost of disregarding the condition $\Delta / d \leq 0.3$ adopted in the numerical simulations. (iii) The higher the number of turns $N$, the higher the measurement sensitivity, but the lower the frequency of the first coil self-resonance (i.e. the lower the upper limit of the measurable frequency band). The coil fabrication quality affects both the measurement accuracy and reproducibility. Indeed, the unperturbed coil impedance must be very stable in order to properly measure the impact of the DUT and reference

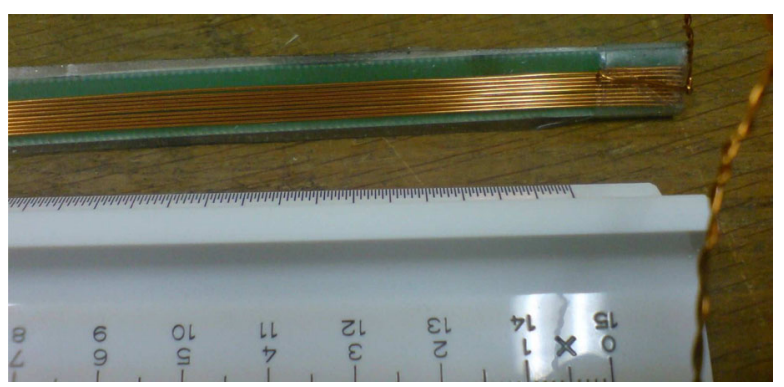

FIG. 10. (Color) Detail of one of the probe coils fabricated for the measurements. materials on the impedance itself. For this reason, the copper windings have been fixed to their rigid support with a thin layer of adhesive plastic tape. A picture of one of the probe coils used for the measurements is shown in Fig. 10.
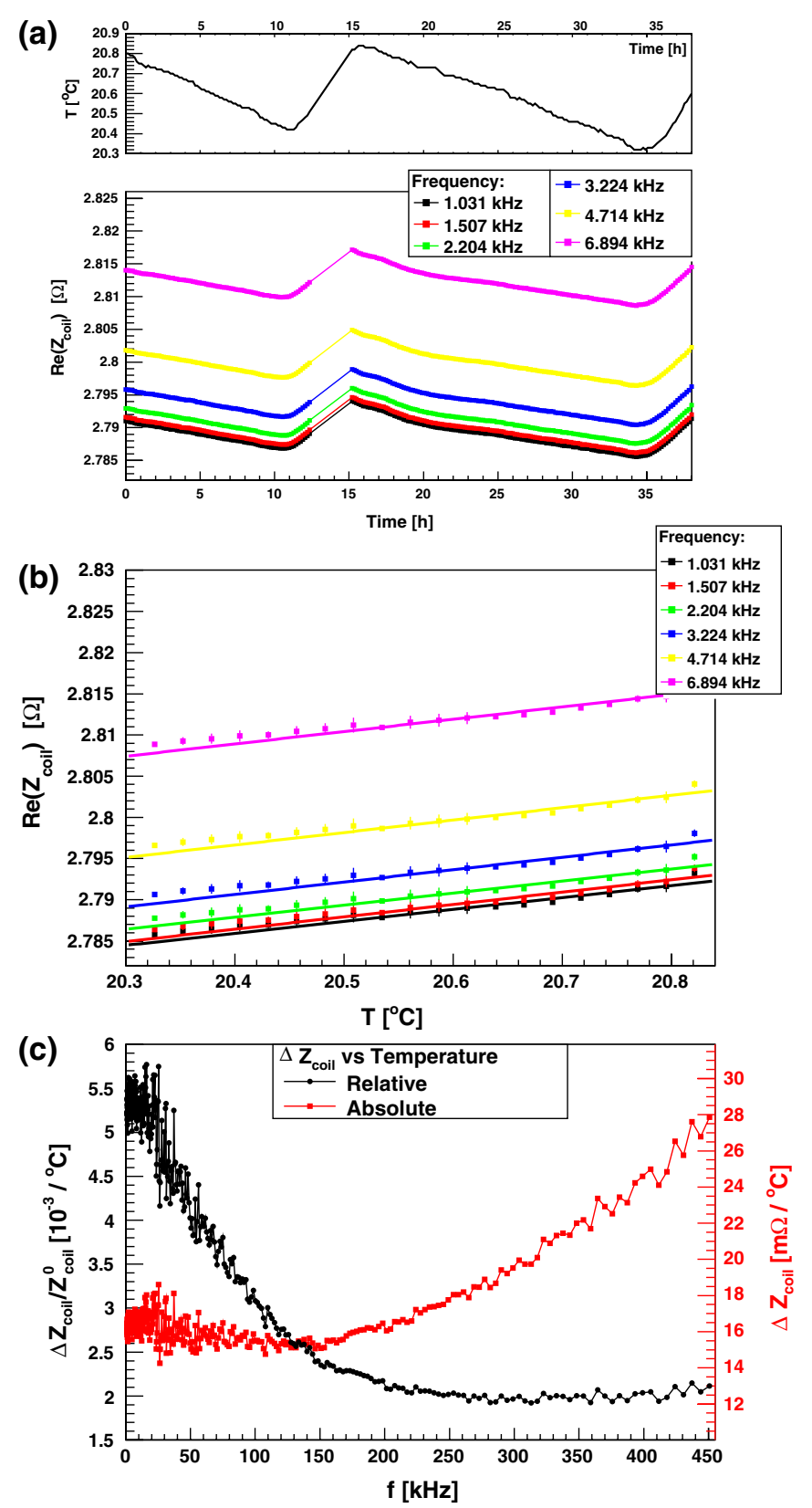

FIG. 11. (Color) Monitoring of temperature effects, while leaving a probe coil inserted between two graphite jaws $(L=1.2 \mathrm{~m}$, gap $=1 \mathrm{~cm})$ for about 38 hours (234 measurements): (a) laboratory temperature (top) and real part of the coil impedance at six frequency samples from 1 to $7 \mathrm{kHz}$ (bottom) as a function of time; (b) real part of the coil impedance as a function of temperature at six frequency samples from 1 to $7 \mathrm{kHz}$. Dots are measurements while the solid lines are the linear fit used to calculate the temperature coefficients below; (c) temperature coefficient as a function of frequency. 
The coil placement and alignment inside the DUT and reference structures are also of primary importance for the accuracy of the results. This was accomplished by using space holders specially manufactured for each coil and DUT geometry. For each set of measurements, we verified that the replacement and realignment of the coil had an impact on the measurement reproducibility smaller than $1 \%$.

The dominant source of uncertainty resulted in a systematic variation of the measured coil impedance with ambient temperature. Since the LCR specifications guarantee a temperature stability much better than the observed variations, the effect was attributed to the variation of coil impedance itself.

We performed a series of measurements with the aim of characterizing such an effect, monitoring the input impedance of one of the fabricated probe coils while leaving it inserted between two stand-alone collimator jaws for many hours. The real part of the measured coil impedance $\operatorname{Re}\left(Z_{\text {coil }}\right)$ is shown in Fig. 11(a) (bottom). Simultaneously, the air temperature was monitored few centimeters apart from the coil, as shown in Fig. 11(a) (top). This plot evidences the good functioning of the air conditioning system that regulated the temperature to a constant value within $0.4^{\circ} \mathrm{C}$. The sawtooth shape is repeated every 24 hours and reflects the laboratory exposure to heat sources that is different during day and night. Despite the efficient temperature control, a variation of $\operatorname{Re}\left(Z_{\text {coil }}\right)$ correlated to temperature is evident, as shown in Fig. 11(b) for six frequencies in the low-frequency range. Figure 11(c) shows the temperature coefficient resulting from the linear fit of the data as taken at several frequency points between $100 \mathrm{~Hz}$ and $450 \mathrm{KHz}$.

Such a temperature dependence is especially critical at low frequencies, for which the difference between the DUT and reference measurements can be of a few $\mathrm{m} \Omega$ as shown

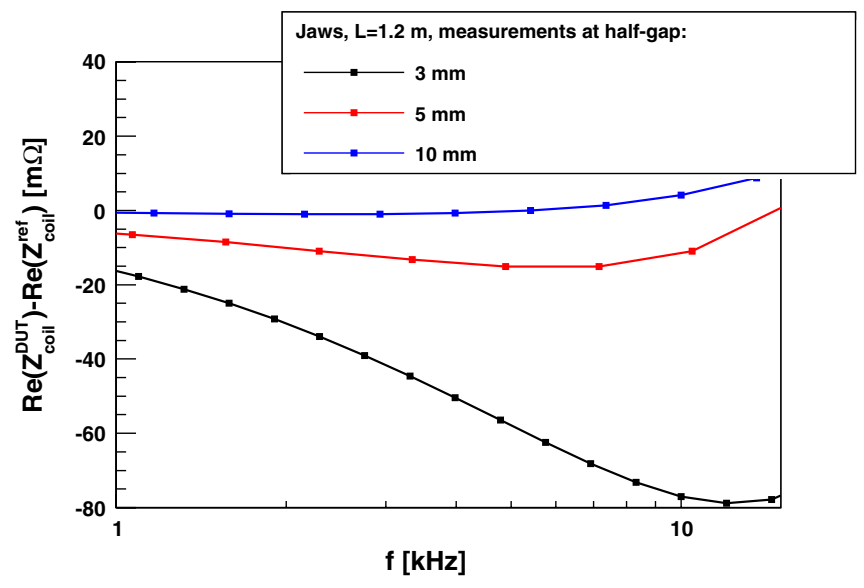

FIG. 12. (Color) Amplitude of the observable $\Delta\left[\operatorname{Re}\left(Z_{\text {coil }}^{\text {DUT }}\right)-\right.$ $\left.\operatorname{Re}\left(Z_{\text {coil }}^{\text {ref }}\right)\right]$ as measured for stand-alone jaws at low frequency for different gaps. The red curve (half-gap $=5 \mathrm{~mm}$ ) corresponds to the same case as the measurements of Fig. 11. in Fig. 12 that refers to measurements with the same coil and geometry as the results of Fig. 11. From Fig. 11(c), it follows that for graphite jaws with an half gap of $5 \mathrm{~mm}$, at $f=10 \mathrm{kHz}$, a temperature variation $\Delta_{T}=0.5^{\circ} \mathrm{C}$ induces a $\Delta \operatorname{Re}\left(Z_{\text {coil }}^{\text {DUT }}\right) \approx 8 \mathrm{~m} \Omega$ that is almost $100 \%$ of the observable $\Delta\left[\operatorname{Re}\left(Z_{\text {coil }}^{\mathrm{DUT}}\right)-\operatorname{Re}\left(Z_{\text {coil }}^{\text {ref }}\right)\right]$ as evident from Fig. 12 . The imaginary part of the coil impedance did not suffer any dependence on temperature.

\section{Measurement results}

For each set of measurements a number of gap (transverse distance between the plates or jaws) values were scanned, in order to compare measurements and theory for different absolute values of the associated transverse impedance. The minimum gap value $g$ was assessed by the condition $g \geq 2 \Delta$, whereas its maximum was determined by the minimum measurable impedance. Measurements with gaps from 5 to $20 \mathrm{~mm}$ were completed, even though only some result examples will be presented in this paper.

\section{Sample plates and stand-alone jaws}

The achieved reproducibility and accuracy can be inferred from the plots in Fig. 13, which reports the results in terms of $\operatorname{Re}\left(Z_{T}^{\text {meas }}\right)$ of measurements performed with sample plates at two different gaps and with two different coils. The experimental results (solid lines) are compared with the correspondent analytical prediction (square dots). Both the reproducibility and the accuracy (in agreement with theory) are well below $1 \%$ down to $f=3 \mathrm{kHz}$ for all measurements apart from the one with the first coil at a collimator half gap of $7 \mathrm{~mm}$ (green plot).

The real part of the measured transverse wall impedance for graphite plates with half gap of $5 \mathrm{~mm}$ is shown in

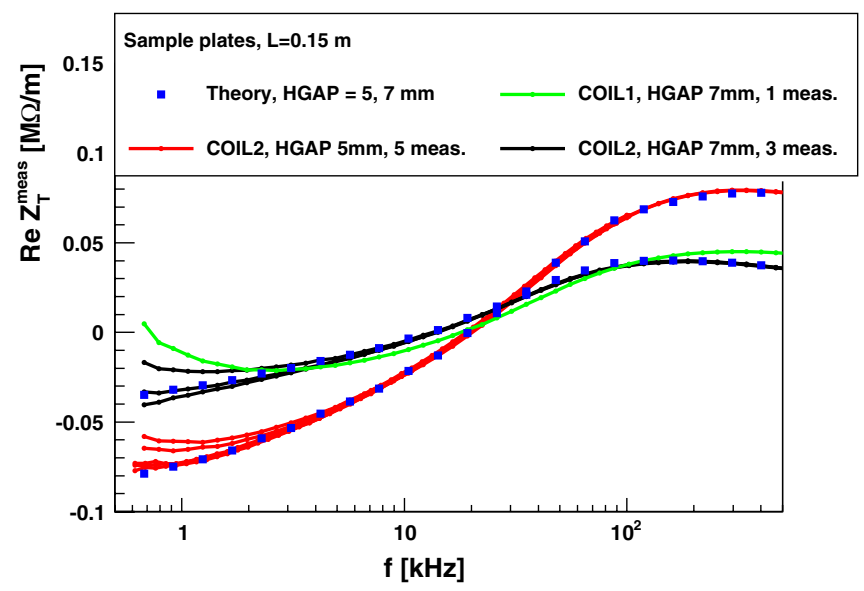

FIG. 13. (Color) Measurement results accuracy and reproducibility using two different coils and two different gaps. The negative values for $f<20 \mathrm{kHz}$ follow from $\operatorname{Re}\left(Z_{W}^{\text {ref }}\right)>$ $\operatorname{Re}\left(Z_{W}^{\mathrm{DUT}}\right)$ at low frequencies, as discussed when defining Eq. (39). 


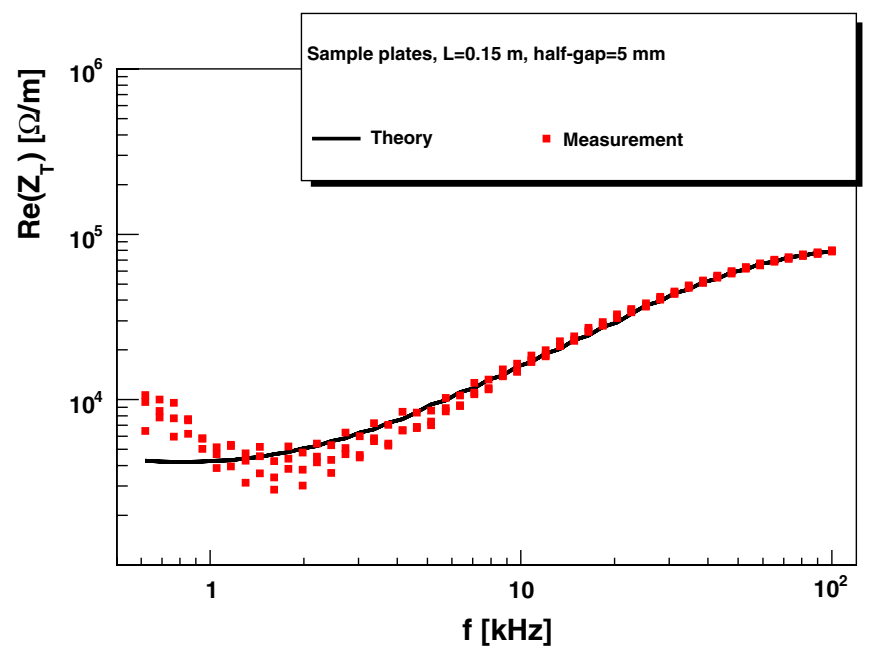

FIG. 14. (Color) Real part of the transverse impedance of graphite plates (stage 1 in Table I).

Fig. 14. This confirms the almost perfect agreement with theory for frequencies above $3 \mathrm{kHz}$.

The results of a series of measurements carried out with stand-alone jaws at different gaps and with different probe coils are presented in Fig. 15. Also in this case the agreement with theory is within $1 \%$, even for large half gaps ( 7 and $10 \mathrm{~mm}$ indicated by the pink and green curves, respectively) corresponding to very small values of $Z_{T}^{\text {meas }}$. This is true for frequencies above $3 \mathrm{kHz}$ and below the first

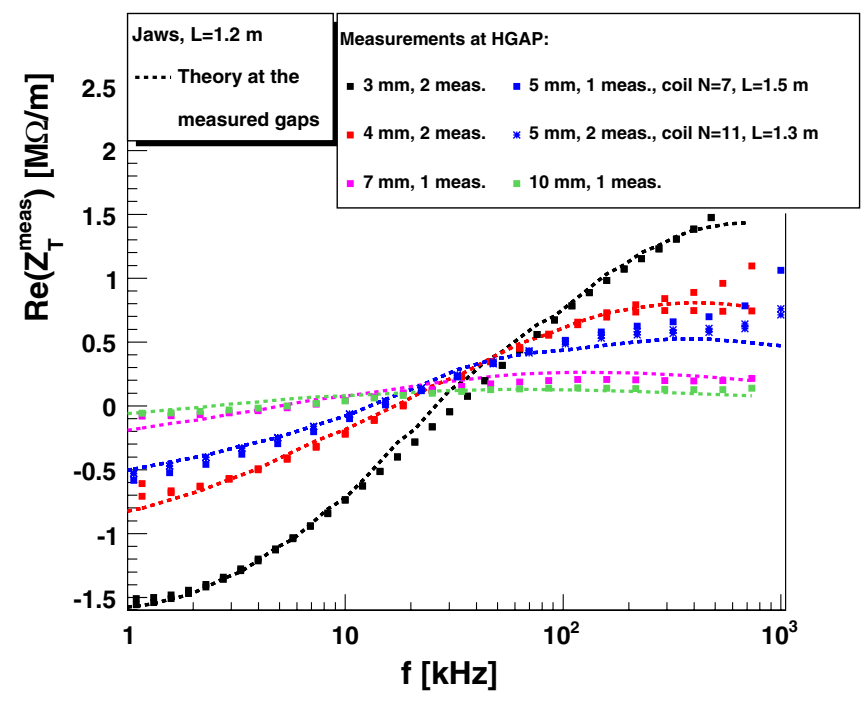

FIG. 15. (Color) Real part of $Z_{T}^{\text {meas }}$, defined in Eq. (38), as measured with stand-alone jaws (stages 2 and 3 in Table I) at different gaps and compared to the correspondent theoretical values. Measurements for half gap of $4 \mathrm{~mm}$ have been taken with two coils differing in length $L$ and number of windings $N$. The negative values for $f<20 \mathrm{kHz}$ follow from $\operatorname{Re}\left(Z_{W}^{\text {ref }}\right)>$ $\operatorname{Re}\left(Z_{W}^{\text {DUT }}\right)$ at low frequencies, as discussed when defining Eq. (39). self-resonance of the coil, which is particularly evident for the blue and red curves at frequencies above $300 \mathrm{kHz}$.

\section{Collimator assembly}

The measurement stages 2 and 3 (see Table I) were meant not only to benchmark the theory, but also to investigate experimentally possible differences in the transverse impedance between stand-alone collimator jaws and their assembly in a collimator. In the latter case the effect of $\mathrm{rf}$ screens and other material surrounding the jaws is very difficult to predict analytically or to simulate. The available jaws and collimator assembly were not fabricated with the same graphite, but this was properly considered in the theoretical predictions. The assembly used for the measurements [see Fig. 8(c)] is a LHC phase 1 collimation prototype (labeled HCTCS_001-CQ000010 [31], of type TCS), representing one of the collimators presently installed in the LHC.

The real and imaginary parts of the transverse impedance for two such configurations are shown in Fig. 16, for a half gap of $4 \mathrm{~mm}$ and using a $2 \mathrm{~m}$ long probe coil with $N=$ 7 and $\Delta=3.25 \mathrm{~mm}$.
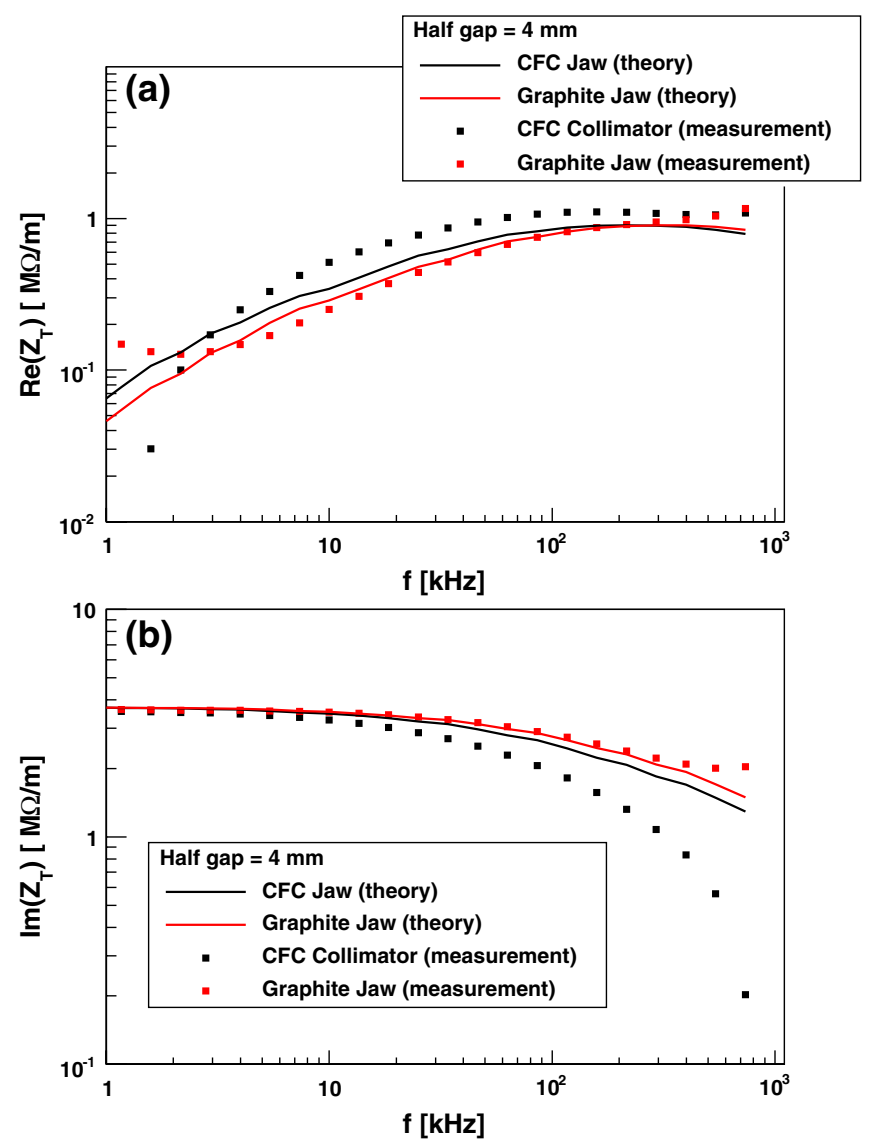

FIG. 16. (Color) (a) Real and (b) imaginary transverse impedance of graphite jaws and CFC collimator assembly (stages 2 and 3 in Table I). 
As indicated in the plot legend, also for the collimator assembly the value predicted with theory refers to the jaws only (geometry and resistivity), since it is very difficult to model the jaws' surroundings. Indeed, the measurement aim is to identify impedance contributions for which theoretical models are not available.

As already discussed above, theory and measurements have a very good agreement for the stand-alone jaws. The agreement is poorer in the case of the collimator assembly, especially for frequencies above $10 \mathrm{kHz}$. Consequently, comparing the measured traces of stages 2 (red dots in the plot) and 3 (black dots), their difference can only be partially attributed to the difference in material resistivity (i.e. the difference between the red and black lines). Dedicated measurements could be desirable to investigate these results in more detail.

Concerning the imaginary part of the transverse impedance, it must be noted that the measurement method responds to magnetic effects only. However, in the lowfrequency range corresponding to the first regime defined in Sec. II, electric field effects are not negligible for the imaginary part. The geometry and material properties for this last set of measurements are such that frequencies above $100 \mathrm{kHz}$ correspond to the second regime for which the measurements are expected to be reliable (i.e. electric effects are negligible). At lower frequencies the agreement with theory must be attributed to the use of Eq. (39): the measured coil impedance $\operatorname{Im}\left(Z_{T}^{\text {meas }}\right)$ in the presence of the DUT is almost zero and the plotted value is dominated by the analytically calculated value $\operatorname{Im}\left(Z_{W}^{\mathrm{ref}}\right)$ that, in this regime, is equal to the theoretical value $\operatorname{Im}\left(Z_{W}^{\text {DUT }}\right)$.

\section{OUTLOOK AND FINAL REMARKS}

A general formalism to compute the transverse wall impedance of an infinitely long multilayer circular beam pipe has been presented. Three frequency regimes are found [10], in agreement with Ref. [12] in the particular case of a (single-layer) LHC collimator. Comparisons with Ref. [5] can be found in Refs. $[9,10]$.

It should be emphasized that a better understanding of the low-frequency regime was reached only very recently, and this is why the term wall impedance in the present manuscript replaces the resistive-wall impedance used in Refs. [1,9-11]. The reason is that at very low frequency, the impedance from the wall (wall impedance) comes only from the electric images (which is the first term of the coherent space-charge impedance) as the ac magnetic images (which is the second term of the coherent spacecharge impedance) disappear. In the reports mentioned above, it was said that the resistive-wall impedance tends to a constant inductive value. However, this constant inductive value should not belong to the resistive-wall impedance and it appeared only because both terms of the coherent space-charge impedance were subtracted from the total impedance (for all frequencies), which is not valid at very low frequency where no ac magnetic images exist. In summary, the final result is the same as in the previous reports [1,9] for $\beta=1$ (and, in particular, Fig. 2) but the term "resistive-wall impedance" is replaced by "wall impedance" (which includes both the effect of the finite resistivity and the coherent space charge). Both impedances coincide at sufficiently high frequencies and high energy, but not at low frequency, where the resistive-wall impedance goes to zero, whereas the wall impedance converges to the impedance of a perfectly conducting beam pipe in dc (i.e. with only electric images).

The measurement campaign aiming at benchmarking novel analytical theories in a low-frequency regime was successful as the measurement results agree within $1 \%$ with theory down to $f=3 \mathrm{kHz}$. This is also confirmed by numerical simulations. The method and the challenges related to the measurements have been discussed. The residual uncertainty at very low frequencies is attributed to thermal effects on the probe coil input impedance: a fraction of degree change in the laboratory temperature induces a coil impedance variation that exceeds the $Z_{\text {coil }}^{\mathrm{DUT}}-Z_{\text {coil }}^{\text {ref }}$ difference at the numerator of Eq. (38).

Even accounting for the effect of the collimator assembly on the total wall impedance, the impact of the collimation system on the total LHC impedance and all the corresponding analyses of beam stabilities discussed in [32] is still valid.

It must be remarked that analytical calculations and numerical simulations refer to infinitely long structures, whereas measurements are obviously performed on devices with finite length. Nevertheless, the general agreement allows considering edge effects as negligible.

On the other hand, it is relevant to stress that numerical simulations and bench measurements with a double-wire (or probe coil) approach excite only the dipolar part of the transverse impedance, as considered in the analytical calculations. The sum of the dipole and quadrupole components can be measured or simulated using a single wire displaced at several off-axis positions. This method has rather poor sensitivity for measurements at low frequencies, and numerical simulations using this approach are planned for future studies.

In addition, the laboratory experiments have the hypothesis that only eddy currents are responsible for the impedance at low frequency and therefore with the probe coil method we neglect the effects of lossless dielectric materials and thus the related imaginary part of the impedance. This is not critical for the cases analyzed here, but measurements of prototype dielectric collimators are difficult and not solved yet.

Finally, it must be outlined that all these studies are already contributing to the design proposals for the LHC phase 2 collimation and provided preliminary results of prototype materials and geometries, which are not yet published. 


\section{ACKNOWLEDGMENTS}

The authors would like to thank O. Aberle, E. Bravin, M. Buzio, R. Chamizo, M. Chanel, A. Grudiev, R. Jones, T. Lefevre, A. Masi, M. Morvillo, J. G. Perez, S. Perrolaz, S. Redaelli, C. Rossi, J. Serrano, all people from the rf workshop, the building 252 team, and many other colleagues from CERN BE, TE, and EN for their help in various phases of the studies. Thanks also to G. Arduini, R. Appleby, R. Assmann, R. Barlow, O. Bruening, S. Chattopadhyay, M. Giovannozzi, R. M. Jones, and L. Rivkin for their support to this work.

\section{APPENDIX: WALL IMPEDANCE FOR NONULTRARELATIVISTIC BEAMS}

In this Appendix, the beam pipe is cylindrical and is composed of a single homogeneous layer extending to infinity (same conditions as in Fig. 2).

Figure 17 shows the real and imaginary parts of the wall impedance as defined in Eq. (20) for different beams for which the relativistic factor $\beta$ is varied from 0.3 to 0.9999 .
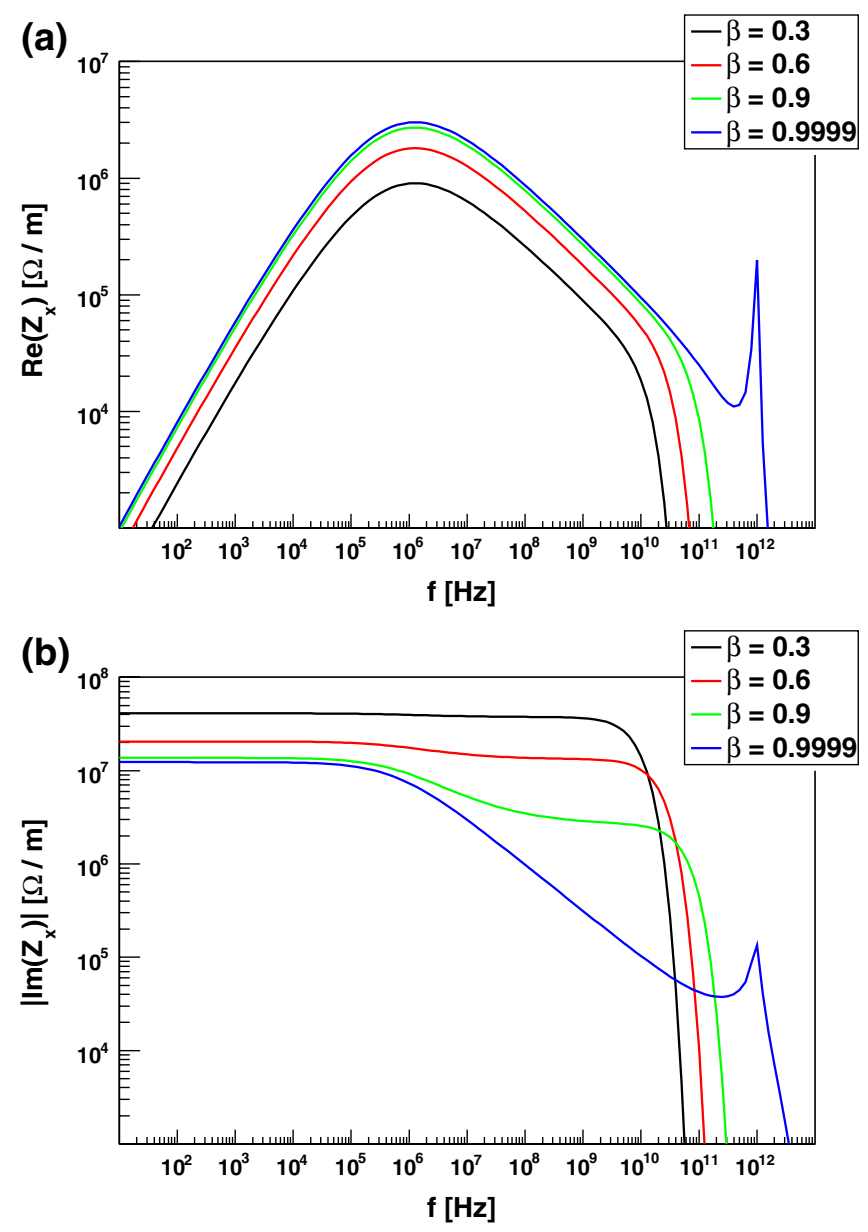

FIG. 17. (Color) (a) Real and (b) imaginary part of the transverse wall impedance for different values of the relativistic factor $\beta$.

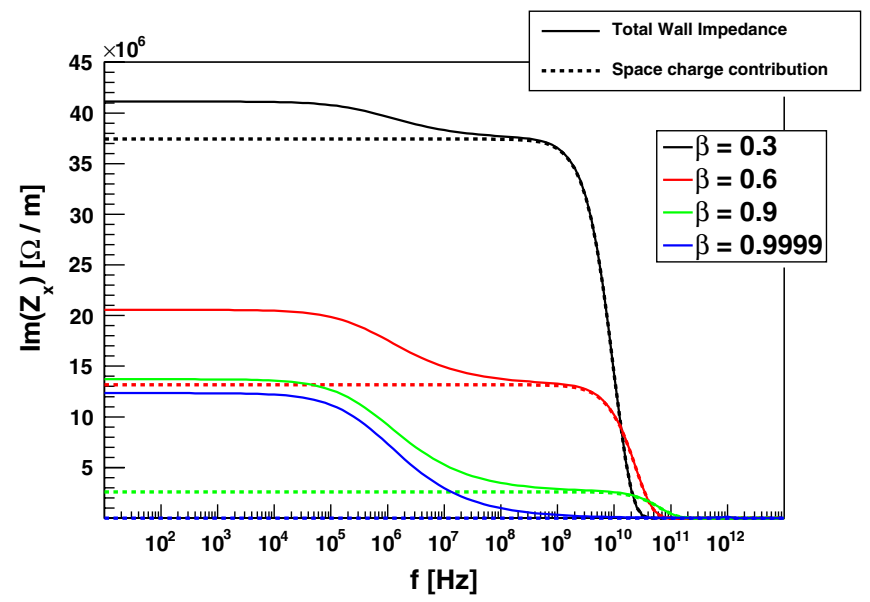

FIG. 18. (Color) Imaginary part of the transverse impedance for different values of $\beta$. For nonrelativistic beams the coherent space-charge contribution dominates.

In Fig. 17(a), the maximum of the real part of the wall impedance is observed to increase gradually with increasing $\beta$. This can be understood if one notices that the real part of the classical thick-wall impedance is proportional to $\beta$. Indeed, the maximum of the real part of the wall impedance lies just at the lower extremity of the frequency range of validity of the classical thick-wall formula, and therefore this maximum also shows a similar increasing behavior with $\beta$.

Furthermore, in Fig. 17(b), the asymptotic value at dc of the imaginary part of the wall impedance decreases with $\beta$. This behavior can be readily understood with Eq. (24).

Finally, it is interesting to notice that the imaginary part of the transverse wall impedance is strongly dominated by the coherent space-charge impedance when the beam is not ultrarelativistic. The imaginary wall impedance and its coherent space-charge contributions are displayed in vertical linear scale in Fig. 18. It can be noticed that the coherent space-charge contribution increases strongly (from negligible to more than 90\%) when the relativistic factor decreases from $\beta=0.999$ to $\beta=0.3$. It can also be noticed that the spectrum of the wall impedance has a lower maximum frequency at low $\beta$.

[1] E. Métral, B. Salvant, and B. Zotter, in Proceedings of the 22nd Particle Accelerator Conference, Albuquerque, 2007 (IEEE, Albuquerque, New Mexico, 2007).

[2] L. Vos, Report No. CERN-AB-2003-005-ABP, 2003.

[3] F. Caspers, U. Iriso-Ariz, and A. Mostacci, in Proceedings of the 20th Particle Accelerator Conference, Portland, OR, 2003 (IEEE, New York, 2003).

[4] J. Laslett, K. Neil, and A. Sessler, Rev. Sci. Instrum. 36, 436 (1965).

[5] A. Burov and V. Lebedev, in Proceedings of the 8th European Particle Accelerator Conference, Paris, 2002 (EPS-IGA and CERN, Geneva, 2002). 
[6] H. Tsutsui, CERN, LHC Project Note 318, 2003.

[7] A. Koschik, F. Caspers, E. Métral, L. Vos, and B. Zotter, in Proceedings of the 9th European Particle Accelerator Conference, Lucerne, 2004 (EPS-AG, Lucerne, 2004).

[8] A. Al-Khateeb, R. W. Hasse, O. Boine-Frankenheim, Wafa M. Daqa, and I. Hofmann, in Proceedings of the 10th European Particle Accelerator Conference, Edinburgh, Scotland, 2006 (EPS-AG, Edinburgh, Scotland, 2006).

[9] B. Zotter, Report No. CERN-AB-2005-043, 2005.

[10] E. Métral, Report No. CERN-AB-2005-084, 2005.

[11] E. Métral, in Proceedings of the 21st Particle Accelerator Conference, Knoxville, 2005 (IEEE, Piscataway, NJ, 2005).

[12] O. Henry and O. Napoly, Report No. CERN-CLIC-Note142-1991, 1991.

[13] J. D. Jackson, Classical Electrodynamics (John Wiley and Sons, New York, 1998), p. 312.

[14] B. Zotter, Report No. ISR-TH-68-58, 1968.

[15] T. Suzuki, Report No. KEK-Preprint-98-127, 1998.

[16] R. L. Gluckstern, Report No. CERN-2000-011, 2000.

[17] K. L. F. Bane, Report No. SLAC/AP-87, 1991.

[18] K. Yokoya, Part. Accel. 41, 221 (1993).

[19] L. J. Laslett, in Proceedings of Summer Study on Storage Rings, Accelerators and Instrumentation at Super High Energies (BNL Report No. 7534, 1963), p. 324.

[20] Ansoft Maxwell®, http://www.ansoft.com/products/em/ maxwell.

[21] T. Kroyer, Report No. CERN-AB-Note-2008-017, 2008.

[22] V. G. Vaccaro, Report No. INFN/TC-94/023, 1994.

[23] G. Nassibian and F. Sacherer, Nucl. Instrum. Methods 159, 21 (1979).
[24] H. Tsutsui, Report No. CERN-SL-Note-2002-034-AP, 2002.

[25] Handbook of Accelerator Physics and Engineering, edited by A. Chao and M. Tinger (World Scientific, Singapore, 1998), p. 570.

[26] Physics of Collective Beams Instabilities in High Energy Accelerators, edited by A. Chao (John Wiley and Sons, New York, 1993), p. 54.

[27] Agilent Technologies®, Product No. HP 8751A, http:// www.home.agilent.com/agilent/facet.jspx?kt=1\&cc= US\&lc=eng\&k=HP+8751A.

[28] Agilent Technologies $\AA$, Product No. 4395A, http:// www.home.agilent.com/agilent/facet.jspx?cc=US\&lc= eng\&k=4395A\&sm=g.

[29] Agilent Technologies $\AA$, Product No. E4980A, http:// www.home.agilent.com/agilent/facet.jspx ?cc=US\&lc= eng\&k=E4980A\&sm=g.

[30] Tektronix ${ }^{\circledR}$, Product No. AD007, http://www2.tek.com/ cmswpt/psdetails.lotr?ct=PS\&cs=psu\&ci=13496\&lc $=E N$.

[31] CERN assembly identifier HCTCS_001-CQ000010, https://edms.cern.ch/equipment/HCTCS_001-CQ000010 (restricted access).

[32] E. Métral, G. Arduini, R. Assmann, A. Boccardi, T. Bohl, C. Bracco, F. Caspers, M. Gasior, O. R. Jones, K. K. Kasinski, T. Kroyer, S. Redaelli, G. Robert-Demolaize, F. Roncarolo, G. Rumolo, B. Salvant, R. Steinhagen, T. Weiler, and F. Zimmermann, in Proceedings of the 22nd Particle Accelerator Conference, Albuquerque, 2007 (Ref. [1]). 